\title{
Recent results from BRAHMS
}

\author{
R. Debbe for the BRAHMS Collaboration \\ Physics Department Brookhaven National Laboratory, Upton, NY, 11973, USA
}

\begin{abstract}
.
The BRAHMS collaboration ended its data collection program in 2006. We are now well advanced in the analysis of a comprehensive set of data that spans systems ranging in mass from $\mathrm{p}+\mathrm{p}$ to $\mathrm{Au}+\mathrm{Au}$ and in energy from $\sqrt{s_{N N}}=62.4$ to 200 $\mathrm{GeV}$. Our analysis has taken two distinct paths: we explore the rapidity dependence of intermediate and high-transverse-momentum, identified-particle production, thus helping to characterize the strongly-interacting quark-gluon plasma (sQGP) formed at RHIC; we also explore particle yields at lower transverse momentum to develop a systematic understanding of bulk particle production at RHIC energies.
\end{abstract}

\section{Introduction}

The time evolution of the hot and dense medium formed at RHIC appears to be well described in terms of the hydrodynamic expansion of a low-viscosity fluid followed by a hadronic cascade [1]. Different aspects of the BRAHMS data can be used to help elucidate the expansion and subsequent hadronization behavior. Comparisons of intermediate and high transverse momentum $\left(p_{T}\right)$ distributions from $\mathrm{A}+\mathrm{A}$ systems to corresponding, properly scaled $\mathrm{p}+\mathrm{p}$ results over a wide range in rapidity contribute to the characterization of the sQGP by highlighting effects that cannot be attributed to the underlying nucleon-nucleon collisions. The study of bulk particle and strangeness production helps to develop the thermodynamical aspects of the reaction. Studying the net proton production as a function of rapidity reveals aspects of the baryon transport in the reaction. This contribution describes recent results from the BRAHMS collaboration.

\section{Energy loss and suppression at high rapidity}

To help frame the discussion, 3+1D hydrodynamic calculations of T. Hirano [2] are used as a guide for understanding some of the observed intermediate- and high- $p_{T}$ results. This model assumes the establishment of a partonic medium that has reached local thermal equilibrium in a particularly short time $(0.6 \mathrm{fm} / \mathrm{c})$, and starts with a maximum energy density of $34.2 \mathrm{GeV} / \mathrm{fm}^{3}$ for $\mathrm{Au}+\mathrm{Au}$ collisons at $200 \mathrm{GeV}$. The bulk properties of that medium are later reflected in the particles produced with $p_{T}<1.5 \mathrm{GeV} / c$. 
Partons generated in hard $2 \rightarrow 2$ QCD interactions obtained from PYTHIA [5] are also part of the calculation to account for the higher $p_{T}$ end of the spectra.

Nuclear effects in A+A collisions can be explored using the Nuclear Modification Factor (NMF). This $p_{T}$ dependent ratio compares the yield of moderate and high $p_{T}$ particles detected from $\mathrm{A}+\mathrm{A}$ system to the ones produced in $\mathrm{p}+\mathrm{p}$ collisions (at $\mathrm{RHIC}$ the measurements can be done at the same energy using the same apparatus) normalized to the Glauber number of binary collisions in the A+A system. A value of this factor close to one is construed as the absence of nuclear effects as the $\mathrm{A}+\mathrm{A}$ collisions are seen as an incoherent sum of $\mathrm{p}+\mathrm{p}$ interactions. This factor was first used in $\mathrm{p}+\mathrm{A}$ studies where it surpassed unity at intermediate values of $p_{T}$ in what is now understood as multiple interactions in the A target. The NMF can also be smaller than one indicating some sort of shadowing, a deficit of scattering points in the initial state, or alternatively reflecting energy loss at partonic level. The NMF has been used to describe earlier $\mathrm{Au}+\mathrm{Au}$ results at RHIC in terms of this energy loss [3. In $\mathrm{d}+\mathrm{Au}$ collisions a clear enhancement at intermediate $p_{T}$ values around mid-rapidity changing into a suppression at high rapidity 44 has been seen as a possible indication of the onset of gluon saturation. The NMFs from central $\mathrm{Au}+\mathrm{Au}$ collisions at $200 \mathrm{GeV}$ measured by BRAHMS show a remarkable feature; the factor is practically unchanged as function of rapidity from $y=0$ to $y=3.1$, see Fig. 11.

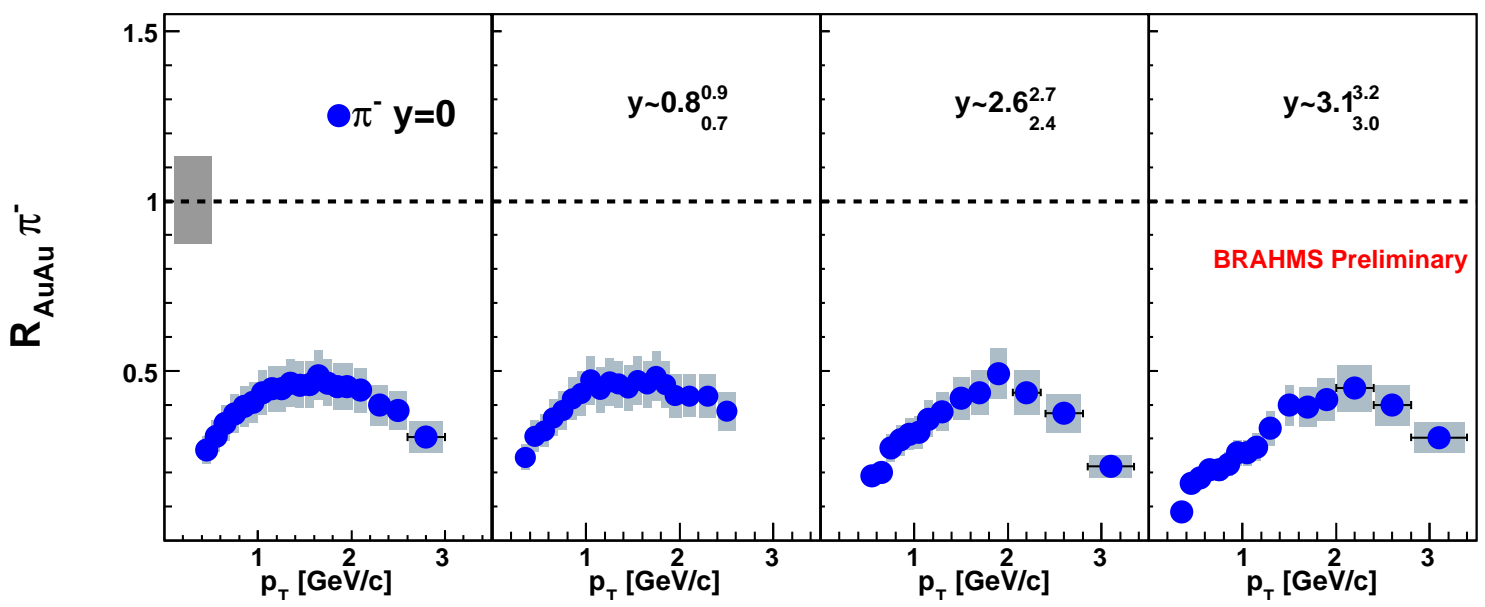

Figure 1. The NMF for negative pion production measured in central $(0-10 \%)$ $\mathrm{Au}+\mathrm{Au}$ collisions at $200 \mathrm{GeV}$. Statistical errors are smaller than the symbols and the estimated systematic errors are displayed as shaded boxes. The estimated error on the number of binary collisions is shown with the gray box centered at 1 .

The hydrodynamical model is used to guide our understanding of the observed weak rapidity dependence of the NMFs obtained from our most central events. The transit of high energy partons through the expanding medium can be followed starting from their generation point. At each step of the hydro calculation the energy of the parton and the local density of the medium are used to calculate the fractional energy loss by gluon emission using the Gyulassy, Levai and Vitev model [6]. After approximately $8 \mathrm{fm} / \mathrm{c}$ the 


\begin{tabular}{|l|c|c|c|c|}
\hline Rapidity & $\mathrm{y}=0$ & $\mathrm{y}=1$ & $\mathrm{y}=2$ & $\mathrm{y}=3$ \\
\hline$\epsilon(0,0)\left[\mathrm{GeV} / \mathrm{fm}^{3}\right]$ at $\tau=0.6 \mathrm{fm} / \mathrm{c}$ & 34.2 & 34.2 & 34.2 & 21.2 \\
$\epsilon(0,0)\left[\mathrm{GeV} / \mathrm{fm}^{3}\right]$ at $\tau=8.4 \mathrm{fm} / \mathrm{c}$ & 5.3 & 5.2 & 4.6 & 2.8 \\
Average fractional energy loss & $17.2 \%$ & $16.9 \%$ & $15.2 \%$ & $10.1 \%$ \\
\hline
\end{tabular}

Table 1. Initial and final energy density at the center of the system at four rapidity values, as well as average energy loss for central $\mathrm{Au}+\mathrm{Au}$ hydro simulations.

system has expanded and cooled to a level where the interactions of the partons with the medium end. The initial and final values of the energy density at each rapidity are listed in Table 1. The average total fractional energy lost by partons is also listed in this table and it shows a drop by almost a factor of 2 as rapidity changes from 0 to 3 .

If one were to naively translate the factor of two drop in energy loss shown in Table 1 into NMF's, one would see a gradual increase from its value at mid-rapidity to values closer to one. However, it should also be noted that spectral shapes do change with rapidity. The increased steepness of the cross sections at high rapidity, resulting from phase space limitations, might offset the reduced energy loss there, keeping the observed suppression constant. Other effects like shadowing or the onset of saturation in the nuclei wave functions at RHIC energies may be contributing factors to the results shown in Fig. 1, The data calls for a detailed theoretical study before firm conclusions can be reached.

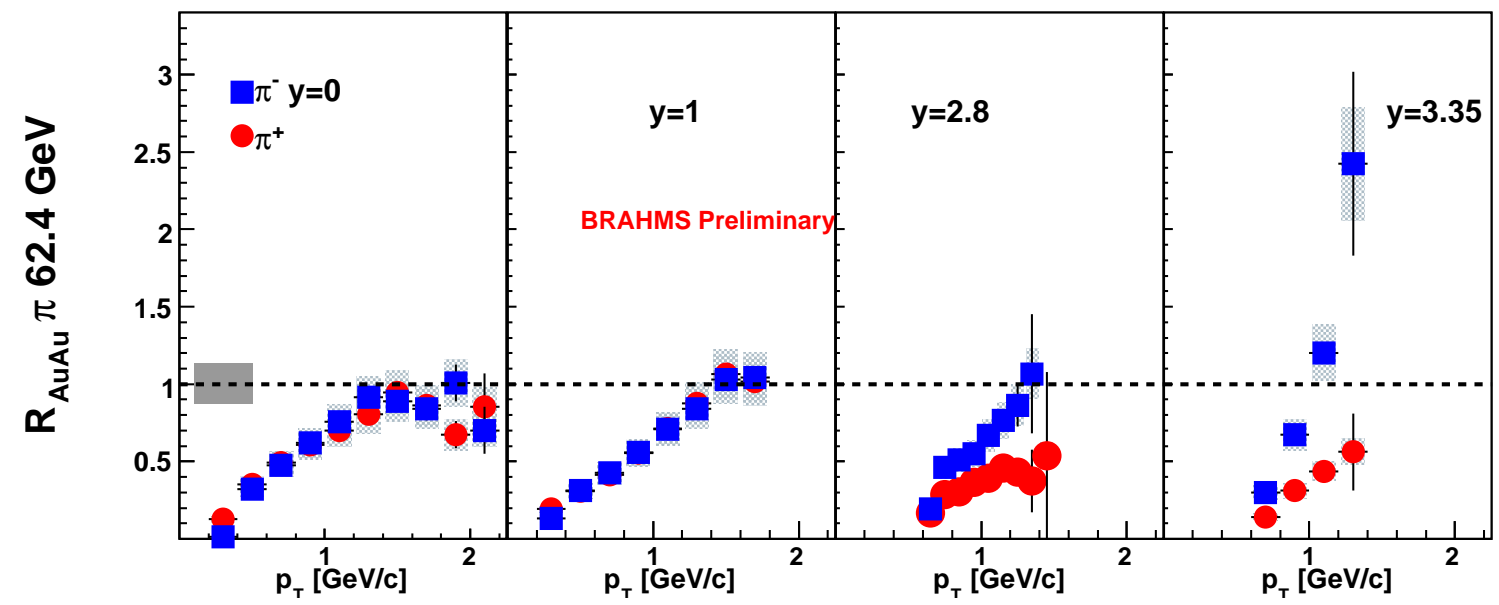

Figure 2. Nuclear Modification factor extracted at four rapidity values for positive and negative pions detected in $0-10 \%$ central $\mathrm{Au}+\mathrm{Au}$ collisions at $62.4 \mathrm{GeV}$. Positive pions are shown with filled circles (red online) and negative pions are shown with filled squares (blue online). Statistical errors are shown with vertical lines at each point, and systematic errors are shown with shaded boxes.

We have also collected and analyzed data at lower energy $(62.4 \mathrm{GeV})$ in $\mathrm{p}+\mathrm{p}$ and $\mathrm{A}+\mathrm{A}$ systems. We report the following results also within the context of a fluid expanding hydrodynamically. Figure 2 shows the NMFs for positive and negative pions 
at four rapidity values. The first striking difference when comparing Fig. 2 to Fig 1 is the completely different rapidity dependence at the two energies. Around mid-rapidity (up to $y \sim 1$ ) the NMF's for positive and negative pions are equal, as if isospin effects are canceled, as was also the case at high energy, but now there is no evidence of a suppression at higher $p_{T}$. At forward rapidities the NMF of negative and positive pions are different and their difference grows more pronounced with $p_{T}$. This difference is related to the isospin of the $\mathrm{p}+\mathrm{p}$ system. According to the hydro calculation at this energy, there is small energy loss at $y=0$ and $y=1$ and practically no energy loss at higher rapidities, well in agreement with what is shown if Fig. 2. More details about BRAHMS high $p_{T}$ analysis can be found in these proceedings [7].

\section{Bulk particle production}

The BRAHMS Collaboration has measured identified particle production in $\mathrm{p}+\mathrm{p}$ and $\mathrm{Au}+\mathrm{Au}$ at $\sqrt{s_{N N}}=62$ and $200 \mathrm{GeV}$. In this section we describe the pion production density in rapidity space, $d n / d y$. Invariant yields for identified particles are constructed from sets of particles detected in the two BRAHMS spectrometers at different angles in narrow rapidity windows. These invariant yields are fully corrected for geometrical acceptance, tracking inefficiencies, and particle identification efficiency as well as losses resulting from multiple scattering, decay in flight and nuclear interactions along the paths in the spectrometers.

We present the yields extracted in $\mathrm{p}+\mathrm{p}$ collision at 200 and $62.4 \mathrm{GeV}$ as well as the centrality-dependent particle densities in $\mathrm{Au}+\mathrm{Au}$ collisions at $200 \mathrm{GeV}$ where we investigate how the size of the colliding system's overlap region affects the production of particles. Total yields are found by integrating over $p_{T}$. Because our measurements do not extend to low values of $p_{T}$ an extrapolation is necessary to evaluate the integral. Several functions were used to fit the $p_{T}$ distributions. The pion distributions from $\mathrm{p}+\mathrm{p}$ collisions at $200 \mathrm{GeV}$ were fitted with the Levy function, the pions at $62.4 \mathrm{GeV}$ are well described by power law shapes around mid-rapidity and by single exponential functions at high rapidity. Finally so called "blast-wave" fits were used to obtain the pion and proton yields in $\mathrm{Au}+\mathrm{Au}$ collisions at $200 \mathrm{GeV}$ shown in the right panel of Fig. 3. The hydrodynamically based blast wave fits included the spectra of pions and protons and their anti-particles at different rapidity values. The velocity profile as a function of the system radius was extracted at mid-rapidity and later fixed for the fits at higher rapidity. The choice of this functional form is motivated by the existence of strong radial flow in $\mathrm{Au}+\mathrm{Au}$ collisions and the very low $p_{T}$ points measured by PHOBOS [10] which exclude functions with strong rise as $p_{T} \rightarrow 0$. Details about similar analyses performed in $\mathrm{p}+\mathrm{p}$ and $\mathrm{d}+\mathrm{Au}$ collisions can be found in these proceedings [9].

The integrated yields are shown in Fig. 3 where we display rapidity densities for

positive pions produced in $\mathrm{p}+\mathrm{p}$ collisions at $\sqrt{s_{N N}}=200$ and $62.4 \mathrm{GeV}$ in the left panel. The right panel shows the same rapidity densities for pions produced in $\mathrm{Au}+\mathrm{Au}$ at 200 $\mathrm{GeV}$ in three centrality samples normalized to the number of participant nucleon pairs 
$N_{\text {part }} / 2$ obtained with a Glauber Monte-Carlo calculation. Within the errors quoted for all of these measurements one interesting feature appears at first glance, all these distributions can be described with single Gaussian shapes. Furthermore, the Gaussian widths follow closely those expected from the Landau-Carruthers hydrodynamical model [8] even though that model makes use of the same equation of state (EOS) with a speed of sound fixed at $c_{s}^{2}=1 / 3$ at all energies and does not include a phase transition. The significance of this agreement is not clear since more sophisticated $1+1 \mathrm{D}$ models which include phase transitions and different EOS have been used to describe successfully the BRAHMS results in central $\mathrm{Au}+\mathrm{Au}$ collisions at $200 \mathrm{GeV}$ and stand in clear conflict with the simple Landau model [11]. It can also be seen in Fig. 3 that the particle density per participating nucleon pair at mid-rapidity are considerably greater in the $\mathrm{A}+\mathrm{A}$ system as compared to the $\mathrm{p}+\mathrm{p}$ system at the same energy. These scaled densities remain almost constant for the different centrality samples of the high energy $\mathrm{Au}+\mathrm{Au}$ collisions.

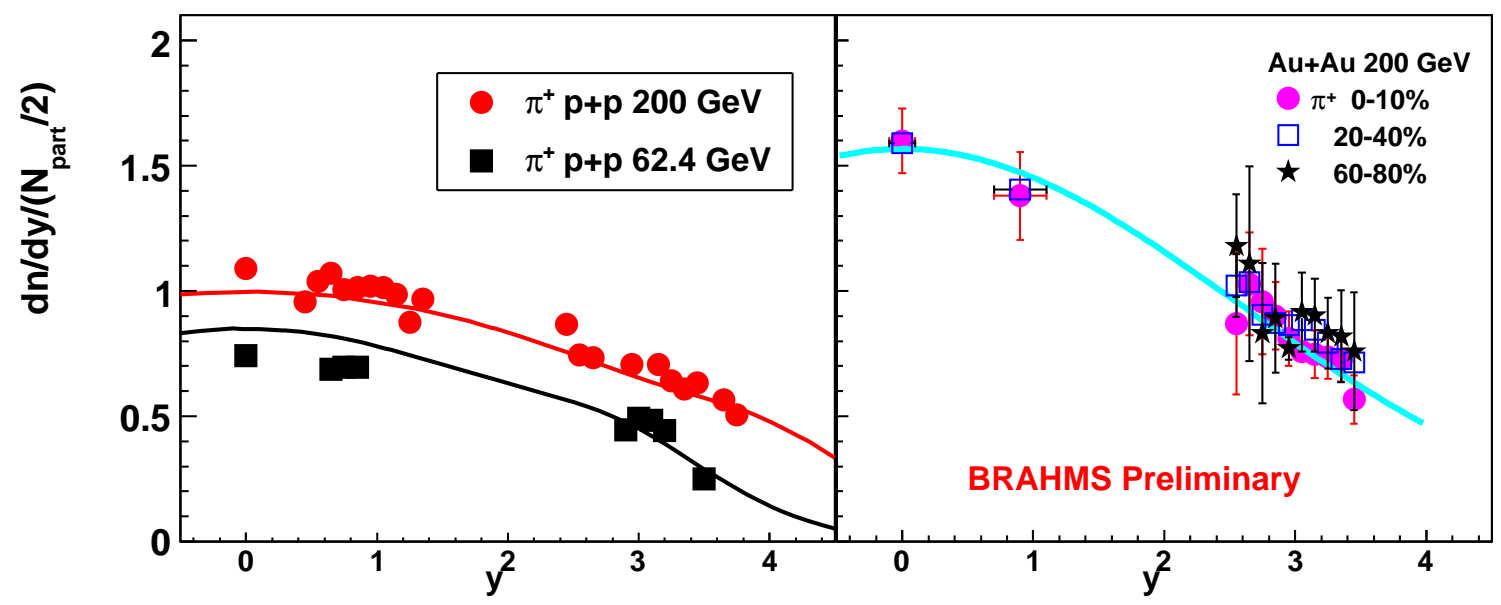

Figure 3. Positive pion densities in rapidity space scaled by the number of participant nucleon pairs. The left panel shows the pions produced in $\mathrm{p}+\mathrm{p}$ collisions at $200 \mathrm{GeV}$ (normalized to total inelastic cross section of $41 \mathrm{mb}$ ) with filled circles (red online) and at $62.4 \mathrm{GeV}$ with black symbols. The smooth curves show the corresponding density obtained with PYTHIA. The panel on the right shows positive pions produced in $\mathrm{Au}+\mathrm{Au}$ at $200 \mathrm{GeV}$ in 5 centrality bins. For clarity, the overall contribution of the spectra statistical and systematical errors as well as the fit uncertainties are displayed for $0-10 \%$ and $60-80 \%$ centrality samples. The smooth curve is a single Gaussian fit to the most central sample.

\section{Strangeness production as function of $\mu_{B}$}

A very interesting correlation is shown in the left panel of Fig. 4: The ratios of antiparticle to particle for kaons and protons yields measured in different $\mathrm{A}+\mathrm{A}$ systems at energies ranging from AGS $(\sim 5 \mathrm{GeV})$, SPS $(9$ to $17 \mathrm{GeV})$ and RHIC lie on a common curve. Moreover, the RHIC points at different rapidities also span the same curve. 
This correlation is well described by statistical models of hadronization [12] and [13] that call for local chemical equilibrium at a common temperature of $170 \mathrm{MeV}$ and a baryochemical potential $\mu_{B}$ in a one to one relation with the anti-proton to proton ratio. Within the same experiment, the value of the baryochemical potential at the late stages of the collisions can be increased if the measurements are made at higher rapidites. In particular, we have studied kaon and proton yields in $\mathrm{Au}+\mathrm{Au}$ collisions at $62.4 \mathrm{GeV}$ where the values of $\mu_{B}$ at the highest measured rapidities are expected to be in the range of those extracted at SPS.
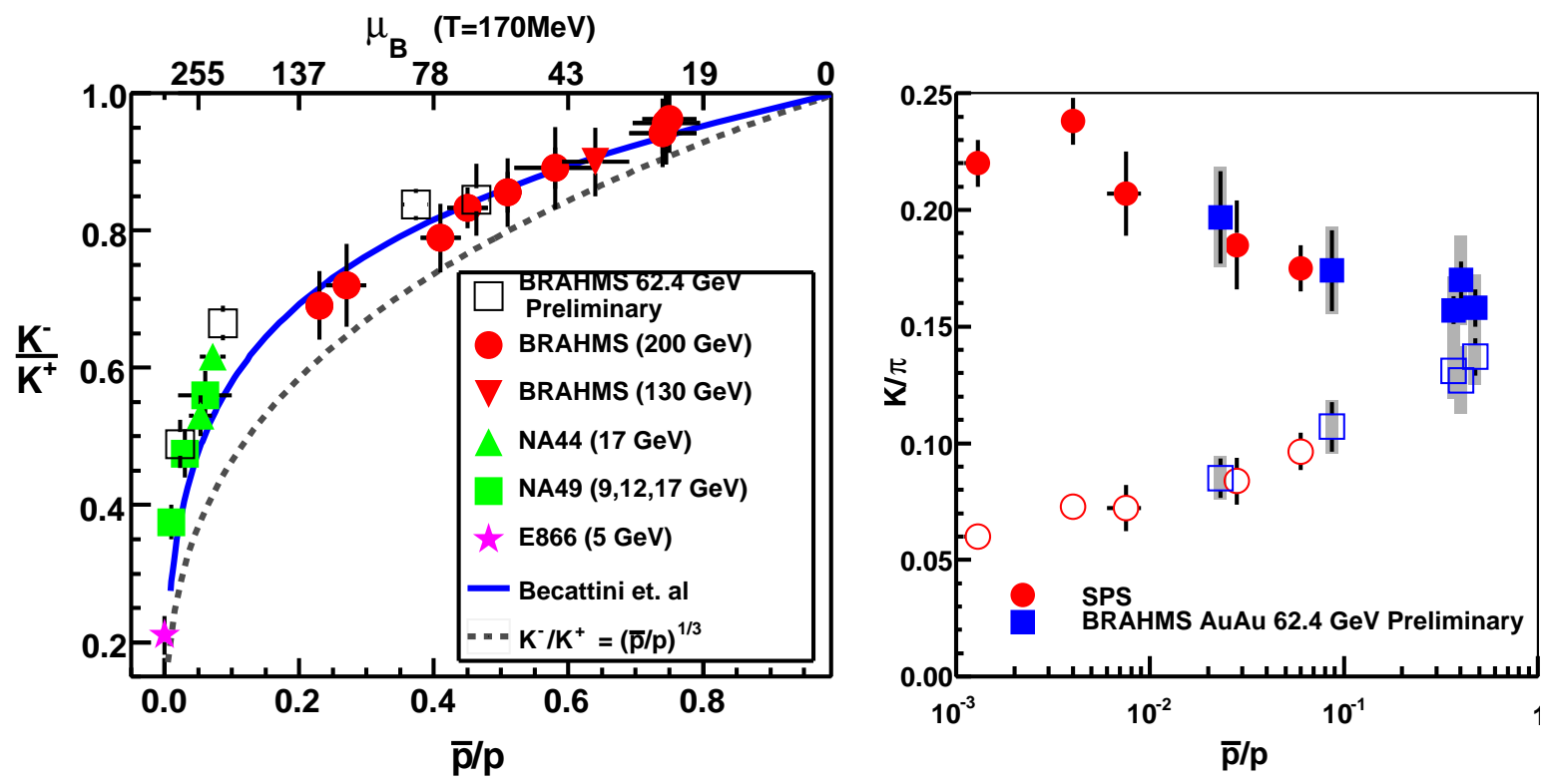

Figure 4. (Left) Anti-particle over particle ratios for kaons and protons in different colliding A+A systems together with a curve based on a statistical model of hadronization. (right) The kaon over pion ratios as function of the anti-proton to proton ratio at the SPS is shown with opened and filled circles (red online) for negative and positive particles respectively. The BRAHMS points extracted from high rapidity $\mathrm{Au}+\mathrm{Au}$ collisions at $62.4 \mathrm{GeV}$ are shown with square symbols (blue online, filled: positive, open: negative). Systematic errors are shown with gray boxes.

The right panel of Fig. 4 shows the ratio of kaon to pion yields, a measure of strangeness production, versus the corresponding anti-proton to proton ratios. The mid-rapidity SPS points shown with open and filled circles (red online) display the high energy side of the so called "Marek Horn" and the BRAHMS results at high rapidity displayed with open and filled squares (blue online) show strikingly similar behavior to the SPS mid-rapidity measurements at their highest energy [14] in what may be considered as an indication of their chemical equivalence. More details on this analysis can be found in these proceedings [15]. 


\section{Baryon transport}

Baryon number is a conserved quantity. Before the collision, all baryons are localized at beam rapidity, but once the collision occurs the total baryon number of the colliding system spreads across rapidity space. The way that rearrangement happens can give great insight into the details of the nuclei-nuclei interaction. The BRAHMS experiment at RHIC, with its ability to cleanly identify protons has collected a large set of data that is now being used to study baryon transport in simple systems like $\mathrm{p}+\mathrm{p}$ collisions at two energies as well as $\mathrm{A}+\mathrm{A}$ collisions. By selecting the centrality of the $\mathrm{A}+\mathrm{A}$ collision one can control the size of the interaction region and extract information on how baryon number transport depends on this geometry. The rapidity distribution of net-proton $(p-\bar{p})$ is used to study how the beam protons are transported in rapidity space. The net-proton distributions, scaled by the number of participating nucleon pairs corresponding to five centrality samples, are displayed in Fig. 5. Our most peripheral events (60-80\%) together with an additional PHENIX point measured at $\mathrm{y}=0$ are shown in the left-most panel of the figure and panels show increasing centrality towards the 0$10 \%$ on the right. The values of net-proton yields have not been corrected for feed-down contributions from weak decays, and the values extracted from previous publications have been scaled back to their uncorrected values. The scaled net-proton distribution for the most peripheral events are similar to the ones extracted from $p+p$ at the same energy; the distribution is strongly peaked in the 3-4 rapidity range. As the volume of the colliding system increases, one can see a clear rearrangement with more protons being shifted all the way to mid-rapidity as the high rapidity peak seen in peripheral events is being transformed into much more rounded shape. This evolution of the net-proton yields with the size of the colliding system, together with a forthcoming distribution extracted from $\mathrm{p}+\mathrm{p}$ collisions as well as low energy $\mathrm{A}+\mathrm{A}$ systems should constitute a data sample that will constrain all baryon transport models.

\section{Summary}

We have contributed to the characterization of the sQGP with our ability to compare the yields of identified particles at intermediate and high $p_{T}$ to appropriately scaled yields of the same particles measured in $\mathrm{p}+\mathrm{p}$ collisions with the same apparatus. At the highest energy our studies of the NMFs of pions at four different rapidity values, together with insights obtained from 3+1D hydrodynamical model lead us to consider the possible presence of phenomena that compensate for a reduced effect of energy loss. We also report on our ongoing work to characterize the systematics of bulk particle production. In particular, we described pion densities in $\mathrm{p}+\mathrm{p}$ and $\mathrm{Au}+\mathrm{Au}$ at 200 and $62.4 \mathrm{GeV}$. All of these distribution display a simple Gaussian shape and appear to scale with the number of participant nucleons. As an aside to our work on bulk particle production we described work done in $\mathrm{Au}+\mathrm{Au}$ at $62.4 \mathrm{GeV}$ to study strangeness production at high rapidity and how it reproduces high energy SPS results. This confirms the fact that 


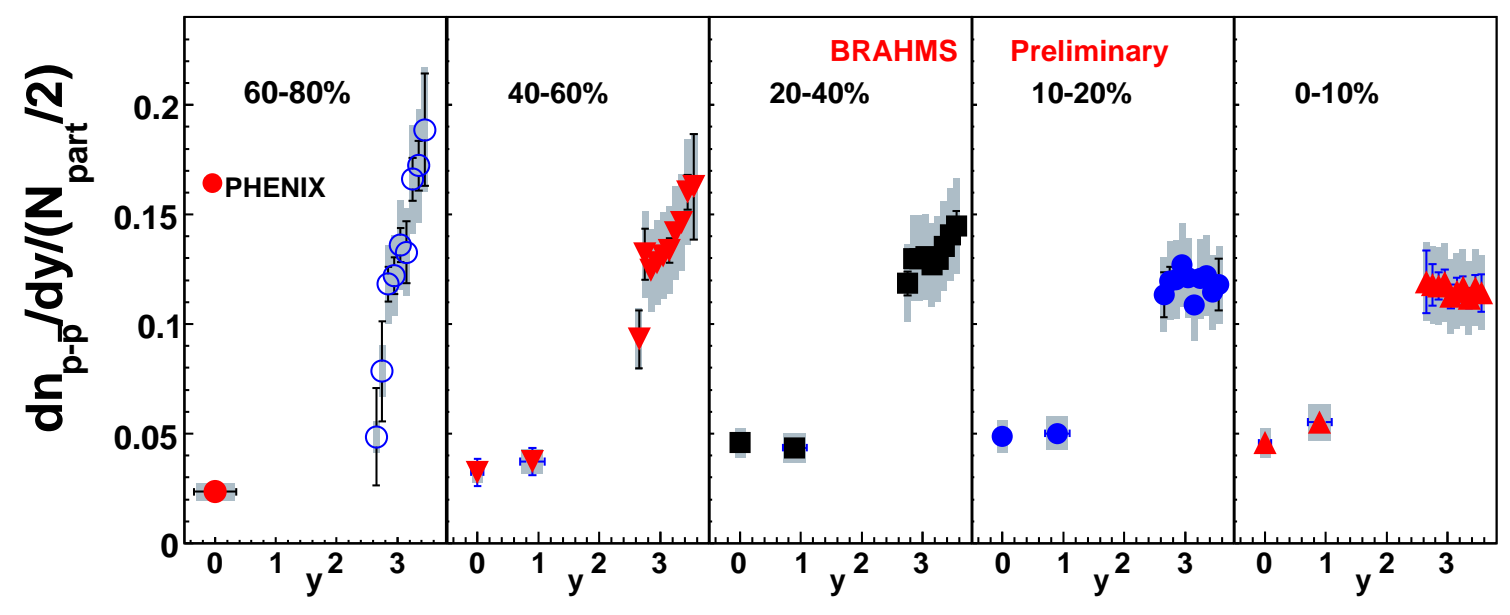

Figure 5. Net proton yields in $\mathrm{Au}+\mathrm{Au}$ collisions at $200 \mathrm{GeV}$ normalized by $N_{\text {part }} / 2$ for different centrality samples. The $y \sim 0$ and $y \sim 1$ points at all centralities were extracted from a previous BRAHMS publication [16] except the single PHENIX point [17] in the $60-80 \%$ centrality sample. No feed down corrections are applied.

in the late stages of the interaction, hadronization proceeds according to an statistical distribution driven mainly by the local value of the baryochemical potential. Finally, we report on our work to establish the baryon transport in $\mathrm{Au}+\mathrm{Au}$ collisions at 200 $\mathrm{GeV}$ studying the distribution of net-protons in rapidity space for samples of events with different centralities. By changing the centrality of the events and with the help of existing transport models, we expect to be able to identify the effect of the interaction volume size on the rates of absorption or re-interaction, as reflected in the yields of protons and anti-protons.

\section{References}

[1] E. Shuryak, these proceedings and references therein.

[2] T.Hirano and Y.Nara, Nucl. Phys. A743, (2004) 305.

[3] I. Arsene et al. Phys. Rev. Lett. 91, 072305 (2003).

[4] I. Arsene et al. Phys. Rev. Lett. 93, 242303 (2004).

[5] T. Sjostrand et al., Comp. Phys. Comm. 135, 238 (2001).

[6] M. Gyulassy, P. Levai, and I. Vitev, Nucl. Phys. B594,371 (2001).

[7] I. Bearden, these proceedings.

[8] P. Carruthers and M. Duong-van, Phys. Rev. D8, 859 (1973).

[9] H. Yang, these proceedings.

[10] B. B. Back et al. Phys. Rev. C70, 051901(R) (2004).

[11] L.M. Satarov, A.V. Merdeev, I.N. Mishustin and H. Stoecker, Phys. Rev. C 75, 024903 (2007).

[12] F. Becattini, J. Cleymans, A. Keranen, E. Suhonen, and K. Redlich, Phys. Rev. C 64, 024901 (2001).

[13] B. Biedroń and W. Broniowski Phys. Rev. C75, 054905 (2007).

[14] NA49 Collaboration, Phys.Rev. C73, 044910 (2006).

[15] I.C. Arsene, these proceedings.

[16] I. Arsene et al. Phys. Rev. C72, 014908 (2005).

[17] S.S. Adler et al. Phys. Rev. C69, 034909 (2004). 